\title{
Boundedness of Littlewood-Paley operators and their commutators on Herz-Morrey spaces with variable exponent
}

\author{
Lijuan Wang and Shuangping Tao*
}

\section{"Correspondence:}

taosp@nwnu.edu.cn

College of Mathematics and

Statistics Science, Northwest

Normal University, Lanzhou, Gansu

730070, P.R. China

\begin{abstract}
The aim of this paper is to establish the vector-valued inequalities for Littlewood-Paley operators, including the Lusin area integrals, the Littlewood-Paley $g$-functions and $g_{\mu}^{*}$-functions, and their commutators on the Herz-Morrey spaces with variable exponent $M \dot{K}_{p, q(\cdot)}^{\alpha, \lambda}\left(\mathbb{R}^{n}\right)$. By applying the properties of $L^{p(\cdot)}\left(\mathbb{R}^{n}\right)$ spaces and the vector-valued inequalities for Littlewood-Paley operators and their commutators generated by $B M O$ function on $L^{p(\cdot)}\left(\mathbb{R}^{n}\right)$, the boundedness of the vector-valued Littlewood-Paley operators and their commutators is obtained on $M \dot{K}_{p, q(\cdot)}^{\alpha, \lambda}\left(\mathbb{R}^{n}\right)$.

MSC: 42B20; 42B25; 42B35
\end{abstract}

Keywords: vector-valued inequality; Littlewood-Paley operators; Herz-Morrey spaces with variable exponent; BMO spaces

\section{Introduction}

Let $\psi \in L^{1}\left(\mathbb{R}^{n}\right)$ and satisfies

(i) $\int_{\mathbb{R}^{n}} \psi(x) d x=0$,

(ii) $|\psi(x)| \leq C(1+|x|)^{-n-\varepsilon}$,

(iii) $|\psi(x+y)-\psi(x)| \leq C|y|^{\gamma}(1+|x|)^{-n-\gamma-\varepsilon},|x| \geq 2|y|$,

where $C, \varepsilon, \gamma$ are all positive constants. Denote $\psi_{t}(x)=t^{-n} \psi(x / t)$ with $t>0$ and $x \in \mathbb{R}^{n}$. Given a function $f \in L_{\mathrm{loc}}^{1}\left(\mathbb{R}^{n}\right)$, the Lusin area integral of $f$ is defined by

$$
S_{\psi, a}(f)(x)=\left(\int_{\Gamma_{a}(x)}\left|\psi_{t} * f(y)\right|^{2} \frac{d y d t}{t^{n+1}}\right)^{1 / 2}
$$

where $\Gamma_{a}(x)$ denote the usual cone of aperture one

$$
\Gamma_{a}(x)=\left\{(t, y) \in \mathbb{R}_{+}^{n+1}:|y-x|<a t, a \geq 1\right\} .
$$

As $a=1$, we denote $S_{\psi, a}(f)$ as $S_{\psi}(f)$.

Now let us turn to introduce the other two Littlewood-Paley operators. It is well known that the Littlewood-Paley operators include also the Littlewood-Paley $g$-functions and the Littlewood-Paley $g_{\mu}^{*}$-functions besides the Lusin area integrals. The Littlewood-Paley $g$-functions, which can be viewed as a 'zero-aperture' version of $S_{\psi}$, and $g_{\mu}^{*}$-functions,

\section{Springer}

○2014 Wang and Tao; licensee Springer. This is an Open Access article distributed under the terms of the Creative Commons Attribution License (http://creativecommons.org/licenses/by/2.0), which permits unrestricted use, distribution, and reproduction in any medium, provided the original work is properly cited. 
which can be viewed as a 'infinite-aperture' version of $S_{\psi}$, are defined, respectively, by

$$
\begin{aligned}
& g(f)(x)=\left(\int_{0}^{\infty}\left|\psi_{t} * f(y)\right|^{2} \frac{d t}{t}\right)^{1 / 2}, \\
& g_{\mu}^{*}(f)(x)=\left(\int_{\mathbb{R}_{+}^{n+1}}\left(\frac{t}{t+|x-y|}\right)^{\mu n}\left|\psi_{t} * f(y)\right|^{2} \frac{d y d t}{t^{n+1}}\right)^{1 / 2}, \quad \mu>0 .
\end{aligned}
$$

If we take $\psi$ to be the poisson kernel, then the functions defined above are the classical Littlewood-Paley operators.

Let $b \in L_{\text {loc }}^{1}\left(\mathbb{R}^{n}\right), m \geq 1$, the corresponding $m$-order commutators of Littlewood-Paley operators above generated by a function $b$ are defined by

$$
\begin{aligned}
& {\left[b^{m}, g_{\psi}\right](f)(x)=\left(\int_{0}^{\infty}\left|\int_{\mathbb{R}^{n}}[b(x)-b(y)]^{m} \psi_{t}(x-y) f(y) d y\right|^{2} \frac{d t}{t}\right)^{1 / 2},} \\
& {\left[b^{m}, S_{\psi, a}\right](f)(x)=\left(\int_{\Gamma_{a}(x)}\left|\int_{\mathbb{R}^{n}}[b(x)-b(z)]^{m} \psi_{t}(y-z) f(z) d z\right|^{2} \frac{d y d t}{t^{n+1}}\right)^{1 / 2}}
\end{aligned}
$$

and

$$
\begin{aligned}
& {\left[b^{m}, g_{\mu}^{*}\right](f)(x)=\left(\int_{\mathbb{R}_{+}^{n+1}}\left(\frac{t}{t+|x-y|}\right)^{\mu n}\left|\int_{\mathbb{R}^{n}}[b(x)-b(z)]^{m} \psi_{t}(y-z) f(z) d z\right|^{2} \frac{d y d t}{t^{n+1}}\right)^{\frac{1}{2}}} \\
& \quad \mu>0 .
\end{aligned}
$$

The Littlewood-Paley operators are very important objects in the study of harmonic analysis. They play very important roles in harmonic analysis and PDE (see [1-3]), so it is natural and meaningful to consider the boundedness of Littlewood-Paley operators and their commutators. Lu and Yang investigated the behavior of Littlewood-Paley operators in the space $\mathrm{CBMO}_{p}\left(\mathbb{R}^{n}\right)$ in [4]. In 2005, Zhang and Liu proved the commutator $\left[b, g_{\psi}\right]$ is bounded on $L^{p}(\omega)$ (see [5]). In 2009, Xue and Ding gave the weighted estimate for Littlewood-Paley operators and their commutators (see [6]). There are some other results about Littlewood-Paley operators in [7-9].

On the other hand, Lebesgue spaces with variable exponent $L^{p(\cdot)}\left(\mathbb{R}^{n}\right)$ become one of the important function spaces due to the fundamental paper [10] by Kováčik and Rákosník. In the past 20 years, the theory of these spaces has made progress rapidly, and the study of it has many applications in fluid dynamics, elasticity, calculus of variations and differential equations with non-standard growth conditions (see [11-15]). In [16], Cruz-Uribe et al. proved the extrapolation theorem which leads the boundedness of some classical operators including the commutator on $L^{p(\cdot)}\left(\mathbb{R}^{n}\right)$. Karlovich and Lerner also independently obtained the boundedness of the singular integral commutators in [17]. Recently, Izuki considered the boundedness of vector-valued sub-linear operators and fractional integrals on Herz-Morrey spaces with variable exponent in [18] and [19], respectively.

Inspired by the above works, in this paper we will consider the vector-valued inequalities of the Littlewood-Paley operators and their $m$-order commutators on Herz-Morrey spaces with variable exponent. To do this, we need recall some definitions about the spaces with variable exponent.

Let $E$ be a Lebesgue measurable set in $\mathbb{R}^{n}$ with measure $|E|>0$. 
Definition 1.1 [10] Let $p(\cdot): E \rightarrow[1, \infty)$ be a measurable function.

The Lebesgue space with variable exponent $L^{p(\cdot)}(E)$ is defined by

$$
L^{p(\cdot)}(E)=\left\{f \text { is measurable }: \int_{E}\left(\frac{|f(x)|}{\eta}\right)^{p(x)} d x<\infty \text { for some constant } \eta>0\right\} .
$$

The space $L_{\text {loc }}^{p(\cdot)}(E)$ is defined by

$$
L_{\text {loc }}^{p(\cdot)}(E)=\left\{f \text { is measurable }: f \in L^{p(\cdot)}(K) \text { for all compact subsets } K \subset E\right\} .
$$

The Lebesgue space $L^{p(\cdot)}(E)$ is a Banach space with the norm defined by

$$
\|f\|_{L^{p(\cdot)}(E)}=\inf \left\{\eta>0: \int_{E}\left(\frac{|f(x)|}{\eta}\right)^{p(x)} d x \leq 1\right\}
$$

We denote

$$
p_{-}:=\operatorname{essinf}\{p(x): x \in E\}, \quad p_{+}:=\operatorname{ess} \sup \{p(x): x \in E\} .
$$

Then $\mathcal{P}(E)$ consists of all $p(\cdot)$ satisfying $p_{-}>1$ and $p_{+}<\infty$.

Let $M$ be the Hardy-Littlewood maximal operator. We denote $\mathcal{B}(E)$ to be the set of all functions $p(\cdot) \in \mathcal{P}(E)$ satisfying the condition that $M$ is bounded on $L^{p(\cdot)}(E)$.

Let $B_{k}=\left\{x \in \mathbb{R}^{n}:|x| \leq 2^{k}\right\}, C_{k}=B_{k} \backslash B_{k-1}, k \in \mathbb{Z}, \chi_{k}=\chi_{C_{k}}$.

Definition 1.2 [18] Let $\alpha \in \mathbb{R}^{n}, 0 \leq \lambda<\infty, 0<p<\infty$, and $q(\cdot) \in \mathcal{P}\left(\mathbb{R}^{n}\right)$. The homogeneous Herz-Morrey spaces with variable exponent $M \dot{K}_{p, q(\cdot)}^{\alpha, \lambda}\left(\mathbb{R}^{n}\right)$ is defined by

$$
M \dot{K}_{p, q(\cdot)}^{\alpha, \lambda}\left(\mathbb{R}^{n}\right)=\left\{f \in L_{\mathrm{loc}}^{q(\cdot)}\left(\mathbb{R}^{n} \backslash\{0\}\right):\|f\|_{M \dot{K}_{p, q(\cdot)}^{\alpha, \lambda}\left(\mathbb{R}^{n}\right)}<\infty\right\}
$$

where

$$
\|f\|_{M \dot{K}_{p, q(\cdot)}^{\alpha, \lambda}\left(\mathbb{R}^{n)}\right)}=\sup _{k_{0} \in \mathbb{Z}} 2^{-k_{0} \lambda}\left\{\sum_{k=-\infty}^{k_{0}} 2^{k \alpha p}\left\|f \chi_{k}\right\|_{L^{q(\cdot)}\left(\mathbb{R}^{n}\right)}^{p}\right\}^{1 / p} .
$$

Remark 1.1 Comparing the homogeneous Herz-Morrey spaces with variable exponent $M \dot{K}_{p, q(\cdot)}^{\alpha, \lambda}\left(\mathbb{R}^{n}\right)$ with the homogeneous Herz spaces with variable exponent $\dot{K}_{q(\cdot)}^{\alpha, p}\left(\mathbb{R}^{n}\right)$ (see $[20])$, where $\dot{K}_{q(\cdot)}^{\alpha, p}\left(\mathbb{R}^{n}\right)$ is defined by

$$
\dot{K}_{q(\cdot)}^{\alpha, p}\left(\mathbb{R}^{n}\right)=\left\{f \in L_{\text {loc }}^{q(\cdot)}\left(\mathbb{R}^{n} \backslash\{0\}\right):\|f\|_{\dot{K}_{q(\cdot)}^{\alpha, p}\left(\mathbb{R}^{n}\right)}=\left(\sum_{k=-\infty}^{\infty} 2^{\alpha k p}\left\|f \chi_{k}\right\|_{L^{q(\cdot)}\left(\mathbb{R}^{n)}\right)}^{p}\right)^{1 / p}<\infty\right\} .
$$

Obviously, $M \dot{K}_{p, q(\cdot)}^{\alpha, 0}\left(\mathbb{R}^{n}\right)=\dot{K}_{q(\cdot)}^{\alpha, p}\left(\mathbb{R}^{n}\right)$.

We now make some conventions. Throughout this paper, given a function $f$, we denote the mean value of $f$ on $E$ by $f_{E}=: \frac{1}{|E|} \int_{E} f(x) d x \cdot p^{\prime}(\cdot)$ means the conjugate exponent of $p(\cdot)$, namely $1 / p(x)+1 / p^{\prime}(x)=1$ holds. $C$ always means a positive constant independent of the main parameters and may change from one occurrence to another. 


\section{Preliminary lemmas}

In this section, we need some conclusions which will be used in the proofs of our main results.

Lemma 2.1 [10] (Generalized Hölder's Inequality) Let $p(\cdot), p_{1}(\cdot), p_{2}(\cdot) \in \mathcal{P}\left(\mathbb{R}^{n}\right)$.

(1) For any $f \in L^{p(\cdot)}\left(\mathbb{R}^{n}\right), g \in L^{p^{\prime} \cdot(\cdot)}\left(\mathbb{R}^{n}\right)$,

$$
\int_{\mathbb{R}^{n}}|f(x) g(x)| d x \leq C_{p}\|f\|_{L^{p(\cdot)}\left(\mathbb{R}^{n}\right)}\|g\|_{L^{p^{\prime}(\cdot)}\left(\mathbb{R}^{n}\right)},
$$

where $C_{p}=1+1 / p_{-}-1 / p_{+}$.

(2) For any $f \in L^{p_{1}(\cdot)}\left(\mathbb{R}^{n}\right), g \in L^{p_{2}(\cdot)}\left(\mathbb{R}^{n}\right)$, when $1 / p(x)=1 / p_{1}(x)+1 / p_{2}(x)$, we have

$$
\begin{aligned}
& \qquad f(x) g(x)\left\|_{L^{p(\cdot)}\left(\mathbb{R}^{n}\right)} \leq C_{p_{1}, p_{2}}\right\| f\left\|_{L^{p_{1}(\cdot)}\left(\mathbb{R}^{n}\right)}\right\| g \|_{L^{p_{2}(\cdot)}\left(\mathbb{R}^{n}\right)}, \\
& \text { where } C_{p_{1}, p_{2}}=\left(1+1 / p_{1_{-}}-1 / p_{1_{+}}\right)^{1 / p_{-}} .
\end{aligned}
$$

Lemma 2.2 [17] If $p(\cdot) \in \mathcal{B}\left(\mathbb{R}^{n}\right)$, then there exist constants $\delta_{1}, \delta_{2}, C>0$, such that for all balls $B \subset \mathbb{R}^{n}$ and all measurable subsets $S \subset B$,

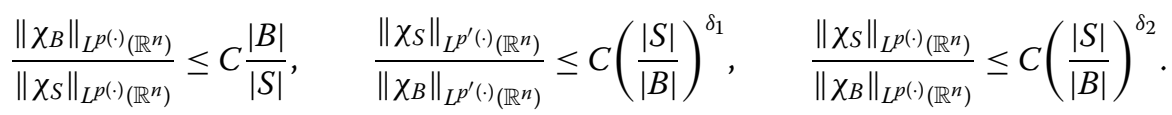

Lemma 2.3 [18] If $p(\cdot) \in \mathcal{B}\left(\mathbb{R}^{n}\right)$, then there exist constants $C>0$, such that for all balls $B \subset \mathbb{R}^{n}$,

$$
\frac{1}{|B|}\left\|\chi_{B}\right\|_{L^{p \cdot(\cdot)}\left(\mathbb{R}^{n}\right)}\left\|\chi_{B}\right\|_{L^{p^{\prime} \cdot(\cdot)\left(\mathbb{R}^{n}\right)}} \leq C .
$$

Lemma 2.4 [20] Let $b \in B M O\left(\mathbb{R}^{n}\right), m$ is a positive integer, there exist constants $C>0$, such that for any $k, j \in \mathbb{Z}$ with $k>j$,

(1) $C^{-1}\|b\|_{*}^{m} \leq \sup _{B} \frac{1}{\left\|\chi_{B}\right\|_{L^{p \cdot(\cdot)}\left(\mathbb{R}^{n}\right)}}\left\|\left(b-b_{B}\right)^{m} \chi_{B}\right\|_{L^{p \cdot(\cdot)}\left(\mathbb{R}^{n}\right)} \leq C\|b\|_{*}^{m}$;

(2) $\left\|\left(b-b_{B_{j}}\right)^{m} \chi_{B_{k}}\right\|_{L^{p \cdot(\cdot)}\left(\mathbb{R}^{n}\right)} \leq C(k-j)^{m}\|b\|_{*}^{m}\left\|\chi_{B_{k}}\right\|_{L^{p(\cdot)}\left(\mathbb{R}^{n)}\right.}$.

Lemma $2.5[21]$ Given an open set $\Omega \subset \mathbb{R}^{n}$, suppose that $p(\cdot) \in \mathcal{P}(\Omega)$ satisfies

$$
\begin{aligned}
& |p(x)-p(y)| \leq \frac{-C}{\ln (|x-y|)}, \quad x, y \in \Omega,|x-y| \leq 1 / 2, \\
& |p(x)-p(y)| \leq \frac{C}{\ln (e+|x|)}, \quad x, y \in \Omega,|y| \geq|x| .
\end{aligned}
$$

Then $p(\cdot) \in \mathcal{B}(\Omega)$, that is, the Hardy-Littlewood maximal operator is bounded on $L^{p(\cdot)}(\Omega)$.

Let $A_{p}$ be the classical Muckenhoupt weighted class.

\section{Lemma 2.6 [16]}

(1) Given a family $\mathcal{F}$ and open set $\Omega \subset \mathbb{R}^{n}$, assume that for some $p_{0}: 1<p_{0}<\infty$, and for every $\omega \in A_{p_{0}}$,

$$
\int_{\Omega} f(x)^{p_{0}} \omega(x) d x \leq C \int_{\Omega} g(x)^{p_{0}} \omega(x) d x, \quad\left(f_{j}, g_{j}\right) \in \mathcal{F} .
$$


Let $p(\cdot) \in \mathcal{P}(\Omega)$ be such that there exists $1<p_{1}<p_{-}$, with $\left(p(\cdot) / p_{1}\right)^{\prime} \in \mathcal{B}(\Omega)$. Then for every $1<q<\infty$ and sequence $\left\{\left(f_{j}, g_{j}\right)\right\}_{j} \in \mathcal{F}$,

$$
\left\|\left(\sum_{j}\left(f_{j}\right)^{q}\right)^{1 / q}\right\|_{L^{p(\cdot)}(\Omega)} \leq C\left\|\left(\sum_{j}\left(g_{j}\right)^{q}\right)^{1 / q}\right\|_{L^{p(\cdot)}(\Omega)} .
$$

(2) If $p(\cdot) \in \mathcal{B}\left(\mathbb{R}^{n}\right)$, then for all $1<q<\infty$,

$$
\left\|\left(\sum_{j}\left(M f_{j}\right)^{q}\right)^{1 / q}\right\|_{L^{p(\cdot)}\left(\mathbb{R}^{n}\right)} \leq C\left\|\left(\sum_{j}\left(\left|f_{j}\right|\right)^{q}\right)^{1 / q}\right\|_{L^{p(\cdot)}\left(\mathbb{R}^{n}\right)} .
$$

Lemma 2.7 [6] Let $\psi \in L^{1}\left(\mathbb{R}^{n}\right)$ satisfies (i)-(iii), $\mu>2,0<\gamma<\min \{(\mu-2) n / 2, \varepsilon\}$, there exists a constant $C$ and for all bounded functions $f$ with compact support:

(1) If $0<p<\infty, \omega \in A_{\infty}$, then

$$
\int_{\mathbb{R}^{n}}\left[g_{\mu}^{*}(f)(x)\right]^{p} \omega(x) d x \leq C[\omega]_{A_{\infty}}^{p} \int_{\mathbb{R}^{n}}[M(f)(x)]^{p} \omega(x) d x .
$$

(2) If $1<p<\infty, \omega \in A_{p}, b \in B M O$, then

$$
\int_{\mathbb{R}^{n}}\left(\left[b^{m}, g_{\mu}^{*}\right](f)(x)\right)^{p} \omega(x) d x \leq C \int_{\mathbb{R}^{n}}|(f)(x)|^{p} \omega(x) d x .
$$

\section{Remark 2.1}

(1) If we replace $g_{\mu}^{*}$ with $S_{\psi, a}$, then the results of Lemma 2.7 also hold. That is due to for any $x \in \mathbb{R}^{n}, \mu \geq 1$, the inequalities $S_{\psi, a}(f)(x) \leq C g_{\mu}^{*}(f)(x)$ and $\left[b^{m}, S_{\psi, a}\right](f)(x) \leq C\left[b^{m}, g_{\mu}^{*}\right](f)(x)$ hold.

(2) According to the argument in [6], we may deduce that Lemma 2.7 is also suit to $g_{\psi}$.

Combining Lemmas 2.6-2.7 and Remark 2.1, we get the following conclusions.

Lemma 2.8 Let $\psi \in L^{1}\left(\mathbb{R}^{n}\right)$ satisfies (i)-(iii). If $p(\cdot) \in \mathcal{B}\left(\mathbb{R}^{n}\right), 1<q<\infty$, then for all bounded compactly support functions $f_{j}$ such that $\left\{f_{j}\right\}_{j=1}^{\infty} \in L^{p(\cdot)}\left(l^{q}\right)$, that is $\left\|\left(\sum_{j}\left(\left|f_{j}\right|\right)^{q}\right)^{1 / q}\right\|_{L^{p \cdot(\cdot)}\left(\mathbb{R}^{n}\right)}<\infty$, the below vector-valued inequalities hold:

(1) $\left\|\left(\sum_{j}\left(S_{\psi, a}(f)_{j}\right)^{q}\right)^{1 / q}\right\|_{L^{p(\cdot)}\left(\mathbb{R}^{n}\right)} \leq C\left\|\left(\sum_{j}\left(\left|f_{j}\right|\right)^{q}\right)^{1 / q}\right\|_{L^{p(\cdot)}\left(\mathbb{R}^{n)}\right)}$,

(2) $\left\|\left(\sum_{j}\left(g_{\psi}(f)_{j}\right)^{q}\right)^{1 / q}\right\|_{L^{p(\cdot)}\left(\mathbb{R}^{n}\right)} \leq C\left\|\left(\sum_{j}\left(\left|f_{j}\right|\right)^{q}\right)^{1 / q}\right\|_{L^{p \cdot(\cdot)}\left(\mathbb{R}^{n}\right)}$,

(3) $\left\|\left(\sum_{j}\left(g_{\mu}^{*}(f)_{j}\right)^{q}\right)^{1 / q}\right\|_{L^{p(\cdot)}\left(\mathbb{R}^{n}\right)} \leq C\left\|\left(\sum_{j}\left(\left|f_{j}\right|\right)^{q}\right)^{1 / q}\right\|_{L^{p(\cdot)}\left(\mathbb{R}^{n}\right)}$,

where $\mu>2,0<\gamma<\min \{(\mu-2) n / 2, \varepsilon\}$.

Lemma 2.9 Let $b \in B M O, \psi \in L^{1}\left(\mathbb{R}^{n}\right)$ satisfies (i)-(iii), $m \in \mathbb{N} \backslash\{0\}$. If $p(\cdot) \in \mathcal{B}\left(\mathbb{R}^{n}\right)$, $1<q<\infty$, then for all bounded compactly support functions $f_{j}$ such that $\left\{f_{j}\right\}_{j=1}^{\infty} \in L^{p(\cdot)}\left(l^{q}\right)$, that is $\left\|\left(\sum_{j}\left(\left|f_{j}\right|\right)^{q}\right)^{1 / q}\right\|_{L^{p \cdot(\cdot)}\left(\mathbb{R}^{n}\right)}<\infty$, the following vector-valued inequalities hold:

(1) $\left\|\left(\sum_{j}\left(\left[b^{m}, S_{\psi, a}\right](f)_{j}\right)^{q}\right)^{1 / q}\right\|_{L^{p \cdot(\cdot)}\left(\mathbb{R}^{n)}\right.} \leq C\left\|\left(\sum_{j}\left(\left|f_{j}\right|\right)^{q}\right)^{1 / q}\right\|_{L^{p(\cdot)}\left(\mathbb{R}^{n)}\right.}$,

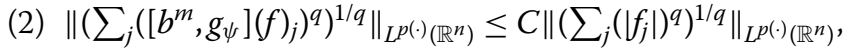

(3) $\left\|\left(\sum_{j}\left(\left[b^{m}, g_{\mu}^{*}\right](f)_{j}\right)^{q}\right)^{1 / q}\right\|_{L^{p(\cdot)}\left(\mathbb{R}^{n}\right)} \leq C\left\|\left(\sum_{j}\left(\left|f_{j}\right|\right)^{q}\right)^{1 / q}\right\|_{L^{p(\cdot)}\left(\mathbb{R}^{n)}\right)}$,

where $\mu>2,0<\gamma<\min \{(\mu-2) n / 2, \varepsilon\}$. 


\section{Main results and their proofs}

In this section, we will establish the vector-valued inequalities of the Littlewood-Paley operators, $S_{\psi, a}, g_{\psi}, g_{\mu}^{*}$, and their $m$-order commutators on Herz-Morrey spaces with variable exponent, respectively.

We begin with the study of the vector-valued inequalities of the Littlewood-Paley operators on $M \dot{K}_{p, q(\cdot)}^{\alpha, \lambda}\left(\mathbb{R}^{n}\right)$.

Theorem 3.1 Suppose that $\psi \in L^{1}\left(\mathbb{R}^{n}\right)$ satisfies (i)-(iii), $q(\cdot) \in \mathcal{B}\left(\mathbb{R}^{n}\right)$. Let $0<p<\infty$, $1<r<\infty, \lambda-n \delta_{2}<\alpha<\lambda+n \delta_{1}$, where $\delta_{1}, \delta_{2}$ is the constant in Lemma 2.2. Then for all function sequences $\left\{f_{h}\right\}_{h=1}^{\infty}:\left\|\left\{\sum_{h}\left|f_{h}\right|^{r}\right\}^{1 / r}\right\|_{M \dot{K}_{p, q(\cdot)}^{\alpha, \lambda}\left(\mathbb{R}^{n}\right)}<\infty$, the following vector-valued inequalities hold:

(1) $\left\|\left\{\sum_{h}\left|S_{\psi, a}\left(f_{h}\right)\right|^{r}\right\}^{1 / r}\right\|_{M \dot{K}_{p, q(\cdot)}^{\alpha, \lambda}\left(\mathbb{R}^{n}\right)} \leq C\left\|\left\{\sum_{h}\left|f_{h}\right|^{r}\right\}^{1 / r}\right\|_{M \dot{K}_{p, q(\cdot)}^{\alpha, \lambda}\left(\mathbb{R}^{n}\right)^{\prime}}$,

(2) $\left\|\left\{\sum_{h}\left|g_{\psi}\left(f_{h}\right)\right|^{r}\right\}^{1 / r}\right\|_{M \dot{K}_{p, q(\cdot)}^{\alpha, \lambda}\left(\mathbb{R}^{n}\right)} \leq C\left\|\left\{\sum_{h}\left|f_{h}\right|^{r}\right\}^{1 / r}\right\|_{M \dot{K}_{p, q(\cdot)}^{\alpha, \lambda}\left(\mathbb{R}^{n}\right)}$,

(3) $\left\|\left\{\sum_{h}\left|g_{\mu}^{*}\left(f_{h}\right)\right|^{r}\right\}^{1 / r}\right\|_{M \dot{K}_{p, q(\cdot)}^{\alpha, \lambda}\left(\mathbb{R}^{n}\right)} \leq C\left\|\left\{\sum_{h}\left|f_{h}\right|^{r}\right\}^{1 / r}\right\|_{M \dot{K}_{p, q(\cdot)}^{\alpha, \lambda}\left(\mathbb{R}^{n}\right)}$,

where $\mu>3+2(\varepsilon+\gamma) / n, 0<\gamma<\min \{(\mu-2) n / 2, \varepsilon\}$, and the constant $C$ is independent of $\left\{f_{h}\right\}_{h=1}^{\infty}$.

Proof Firstly, we consider the inequality (1). For any function sequence $\left\{f_{h}\right\}_{h}$ satisfies \|\|$\left\{f_{h}\right\}_{h}\left\|_{l^{r}}\right\|_{M \dot{K}_{p, q(\cdot)}^{\alpha, \lambda}\left(\mathbb{R}^{n}\right)}<\infty$, we write

$$
f_{h}(x)=\sum_{j=-\infty}^{\infty} f_{h}(x) \chi_{j}(x) \triangleq \sum_{j=-\infty}^{\infty} f_{h}^{j}(x) .
$$

Thus

$$
\begin{aligned}
& \left\|\left\{\sum_{h}\left|S_{\psi, a}\left(f_{h}\right)\right|^{r}\right\}^{1 / r}\right\|_{M \dot{K}_{p, q(\cdot)}^{\alpha, \lambda}\left(\mathbb{R}^{n}\right)} \\
& =\sup _{k_{0} \in \mathbb{Z}} 2^{-k_{0} \lambda}\left\{\sum_{k=-\infty}^{k_{0}} 2^{k \alpha p}\left\|\left\{\sum_{h}\left|\sum_{j=-\infty}^{\infty} S_{\psi, a}\left(f_{h}^{j}\right)\right|^{r}\right\}^{1 / r} \chi_{k}\right\|_{L^{q(\cdot)}\left(\mathbb{R}^{n}\right)}^{p}\right\}^{1 / p} \\
& \leq \sup _{k_{0} \in \mathbb{Z}} 2^{-k_{0} \lambda}\left\{\sum_{k=-\infty}^{k_{0}} 2^{k \alpha p}\left(\sum_{j=-\infty}^{\infty}\left\|\left\{\sum_{h}\left|S_{\psi, a}\left(f_{h}^{j}\right)\right|^{r}\right\}^{1 / r} \chi_{k}\right\|_{L^{q(\cdot) \cdot\left(\mathbb{R}^{n}\right)}}\right)^{p}\right\}^{1 / p} \\
& \leq C \sup _{k_{0} \in \mathbb{Z}} 2^{-k_{0} \lambda}\left\{\sum_{k=-\infty}^{k_{0}} 2^{k \alpha p}\left(\sum_{j=-\infty}^{k-3}\left\|\left\{\sum_{h}\left|S_{\psi, a}\left(f_{h}^{j}\right)\right|^{r}\right\}^{1 / r} \chi_{k}\right\|_{L^{q(\cdot)}\left(\mathbb{R}^{n}\right)}\right)^{p}\right\}^{1 / p} \\
& +C \sup _{k_{0} \in \mathbb{Z}} 2^{-k_{0} \lambda}\left\{\sum_{k=-\infty}^{k_{0}} 2^{k \alpha p}\left(\sum_{j=k-2}^{k+2}\left\|\left\{\sum_{h}\left|S_{\psi, a}\left(f_{h}^{j}\right)\right|^{r}\right\}^{1 / r} \chi_{k}\right\|_{L^{q(\cdot)}\left(\mathbb{R}^{n}\right)}\right)^{p}\right\}^{1 / p} \\
& +C \sup _{k_{0} \in \mathbb{Z}} 2^{-k_{0} \lambda}\left\{\sum_{k=-\infty}^{k_{0}} 2^{k \alpha p}\left(\sum_{j=k+3}^{\infty}\left\|\left\{\sum_{h}\left|S_{\psi, a}\left(f_{h}^{j}\right)\right|^{r}\right\}^{1 / r} \chi_{k}\right\|_{L^{q(\cdot)\left(\mathbb{R}^{n}\right)}}\right)^{p}\right\}^{1 / p} \\
& \triangleq D_{1}+D_{2}+D_{3} \text {. }
\end{aligned}
$$


For the term $D_{2}$, noting that $\operatorname{supp} f_{h}^{j} \subset C_{j}$, we can easily obtain, by Lemma 2.8 ,

$$
\begin{aligned}
D_{2} & \leq C \sup _{k_{0} \in \mathbb{Z}} 2^{-k_{0} \lambda}\left\{\sum_{k=-\infty}^{k_{0}} 2^{k \alpha p}\left(\sum_{j=k-2}^{k+2}\left\|\left\{\sum_{h}\left|f_{h}^{j}\right|^{r}\right\}^{1 / r}\right\|_{L^{q(\cdot)\left(\mathbb{R}^{n}\right)}}\right)^{p}\right\}^{1 / p} \\
& \leq C \sup _{k_{0} \in \mathbb{Z}} 2^{-k_{0} \lambda}\left\{\sum_{k=-\infty}^{k_{0}} 2^{k \alpha p}\left\|\left\{\sum_{h}\left|f_{h}\right|^{r}\right\}^{1 / r} \chi_{k}\right\|_{L^{q(\cdot)}\left(\mathbb{R}^{n)}\right)}^{p}\right\}^{1 / p} \\
& =C\left\|\left\{\sum_{h}\left|f_{h}\right|^{r}\right\}^{1 / r}\right\|_{M \dot{K}_{p, q(\cdot)}^{\alpha, \lambda}\left(\mathbb{R}^{n}\right)} .
\end{aligned}
$$

We now turn to estimate $D_{1}$. Let $\Gamma^{\prime}=\left\{y \in \mathbb{R}^{n}:|y-x|<a t, y \leq 2^{j+1}\right\}, \Gamma^{\prime \prime}=\left\{y \in \mathbb{R}^{n}:|y-x|<\right.$ $\left.a t, y>2^{j+1}\right\}$. We write

$$
\begin{aligned}
S_{\psi, a}\left(f_{h}^{j}\right)(x)= & \left(\int_{\Gamma_{a}(x)}\left|\int_{\mathbb{R}^{n}} t^{-n} \psi\left(\frac{y-z}{t}\right) f_{h}^{j}(z) d z\right|^{2} \frac{d y d t}{t^{n+1}}\right)^{1 / 2} \\
\leq & \left(\int_{0}^{\infty} \int_{\Gamma^{\prime}}\left|\int_{\mathbb{R}^{n}} t^{-n} \psi\left(\frac{y-z}{t}\right) f_{h}^{j}(z) d z\right|^{2} \frac{d y d t}{t^{n+1}}\right)^{1 / 2} \\
& +\left(\int_{0}^{\infty} \int_{\Gamma^{\prime \prime}}\left|\int_{\mathbb{R}^{n}} t^{-n} \psi\left(\frac{y-z}{t}\right) f_{h}^{j}(z) d z\right|^{2} \frac{d y d t}{t^{n+1}}\right)^{1 / 2} \\
\triangleq & I+I I .
\end{aligned}
$$

For $I$, observe that as $a \geq 1, x \in C_{k}, j \leq k-3, z \in C_{j}$, then we have $t>\frac{|x-y|}{a} \geq \frac{|x|-|y|}{a}>$ $\frac{2^{k-1}-2^{j+1}}{a} \geq \frac{2^{j+1}}{a}$, and $t+|y-z| \geq \frac{|x-y|}{a}+|y-z| \geq \frac{|x-y|+|y-z|}{a} \geq \frac{|x|-|z|}{a} \geq \frac{3|x|}{4 a}$. Hence, it follows from the condition (ii) that

$$
\begin{aligned}
I & \leq C\left\{\int_{\frac{2^{j+1}}{a}}^{\infty} \int_{\Gamma^{\prime}}\left(\int_{\mathbb{R}^{n}}\left|f_{h}^{j}(z)\right| t^{-n}\left(1+\frac{|y-z|}{t}\right)^{-n-\varepsilon} d z\right)^{2} \frac{d y d t}{t^{n+1}}\right\}^{1 / 2} \\
& \leq C\left\{\int_{\frac{2^{j+1}}{a}}^{\infty} \int_{\Gamma^{\prime}}\left(\int_{\mathbb{R}^{n}}\left|f_{h}^{j}(z)\right| \frac{t^{\varepsilon}}{(t+|y-z|)^{n+\varepsilon}} d z\right)^{2} \frac{d y d t}{t^{n+1}}\right\}^{1 / 2} \\
& \leq C\left(\frac{4 a}{3|x|}\right)^{n}\left\{\int_{\frac{j^{j+1}}{a}}^{\infty} t^{-n-1} d t \int_{|y|<2^{j+1}} d y\right\}^{1 / 2}\left\|f_{h}^{j}\right\|_{L^{1}\left(\mathbb{R}^{n}\right)} \\
& \leq C a^{3 n / 2}|x|^{-n}\left\|f_{h}^{j}\right\|_{L^{1}\left(\mathbb{R}^{n}\right)^{.}}
\end{aligned}
$$

For the estimate of $I I$, denote

$$
\begin{aligned}
I I \leq & C\left\{\int_{0}^{\infty} \int_{\Gamma^{\prime \prime}}\left(\int_{\mathbb{R}^{n}}\left|f_{h}^{j}(z)\right| t^{-n}\left|\psi\left(\frac{y-z}{t}\right)-\psi\left(\frac{y}{t}\right)\right| d z\right)^{2} \frac{d y d t}{t^{n+1}}\right\}^{1 / 2} \\
& +C\left\{\int_{0}^{\infty} \int_{\Gamma^{\prime \prime}}\left(\int_{\mathbb{R}^{n}}\left|f_{h}^{j}(z)\right| t^{-n}\left|\psi\left(\frac{y}{t}\right)\right| d z\right)^{2} \frac{d y d t}{t^{n+1}}\right\}^{1 / 2} \\
\triangleq & I I_{1}+I I_{2} .
\end{aligned}
$$


Wang and Tao Journal of Inequalities and Applications 2014, 2014:227

Page 8 of 17

http://www.journalofinequalitiesandapplications.com/content/2014/1/227

Noting that $y>2^{j+1} \geq 2|z|$, by the condition (iii), we have

$$
\begin{aligned}
I I_{1} \leq & C\left\{\int_{0}^{\infty} \int_{\Gamma^{\prime \prime}}\left(\int_{\mathbb{R}^{n}}\left|f_{h}^{j}(z)\right| t^{-n}\left(\frac{|z|}{t}\right)^{\gamma}\left(1+\frac{|y|}{t}\right)^{-n-\gamma-\varepsilon} d z\right)^{2} \frac{d y d t}{t^{n+1}}\right\}^{1 / 2} \\
\leq & C\left\{\int_{0}^{\infty} \int_{\Gamma^{\prime \prime}} \frac{t^{2 \varepsilon-n-1}}{(t+|y|)^{2(n+\gamma+\varepsilon)}} d y d t\right\}^{1 / 2} \int_{\mathbb{R}^{n}}\left|f_{h}^{j}(z)\right| \cdot|z|^{\gamma} d z \\
\leq & C 2^{j \gamma}\left\{\int_{0}^{|x|} \int_{\Gamma^{\prime \prime}} \frac{t^{2 \varepsilon-n-1}}{(t+|y|)^{2(n+\gamma+\varepsilon)}} d y d t\right. \\
& \left.+\int_{|x|}^{\infty} \int_{\Gamma^{\prime \prime}} \frac{t^{2 \varepsilon-n-1}}{(t+|y|)^{2(n+\gamma+\varepsilon)}} d y d t\right\}^{1 / 2}\left\|f_{h}^{j}\right\|_{L^{1}\left(\mathbb{R}^{n}\right)^{.}}
\end{aligned}
$$

Since $t+|y|>\frac{|x-y|}{a}+|y| \geq \frac{|x-y|+|y|}{a} \geq \frac{|x|}{a}$, then we obtain

$$
\begin{aligned}
& \int_{0}^{|x|} \int_{\Gamma^{\prime \prime}} \frac{t^{2 \varepsilon-n-1}}{(t+|y|)^{2(n+\gamma+\delta)}} d y d t \\
& \leq C\left(\frac{a}{|x|}\right)^{2(n+\gamma+\varepsilon)} \int_{0}^{|x|} \int_{|y-x|<a t} t^{2 \varepsilon-n-1} d y d t \\
& \leq C\left(\frac{a}{|x|}\right)^{2(n+\gamma+\varepsilon)} \int_{0}^{|x|} t^{2 \delta-n-1} a^{n} t^{n} d t \\
& \leq C a^{3 n+2 \gamma+2 \varepsilon}|x|^{-2(n+\gamma)}
\end{aligned}
$$

and

$$
\begin{aligned}
& \int_{|x|}^{\infty} \int_{\Gamma^{\prime \prime}} \frac{t^{2 \varepsilon-n-1}}{(t+|y|)^{2(n+\gamma+\delta)}} d y d t \\
& \quad \leq C \int_{|x|}^{\infty} \int_{|y-x|<a t} t^{-3 n-2 \gamma-1} d y d t \\
& \quad \leq C a^{n} \int_{|x|}^{\infty} t^{-2 n-2 \gamma-1} d y d t \leq C a^{n}|x|^{-2(n+\gamma)} .
\end{aligned}
$$

Thus, we have

$$
I I_{1} \leq C a^{3 n / 2+\gamma+\varepsilon}|x|^{-(n+\gamma)} 2^{j \gamma}\left\|f_{h}^{j}\right\|_{L^{1}\left(\mathbb{R}^{n}\right)} \leq C a^{3 n / 2+\gamma+\varepsilon}|x|^{-n}\left\|f_{h}^{j}\right\|_{L^{1}\left(\mathbb{R}^{n}\right)^{.}}
$$

On the other hand, by the condition (ii) and $t+|y|>\frac{|x|}{a}$, we deduce

$$
\begin{aligned}
I I_{2} \leq & C\left\{\int_{0}^{\infty} \int_{\Gamma^{\prime \prime}}\left(\int_{\mathbb{R}^{n}}\left|f_{h}^{j}(z)\right| t^{-n}\left(1+\frac{|y|}{t}\right)^{-n-\varepsilon} d z\right)^{2} \frac{d y d t}{t^{n+1}}\right\}^{1 / 2} \\
\leq & C\left\{\int_{0}^{\infty} \int_{\Gamma^{\prime \prime}} \frac{t^{2 \varepsilon-n-1}}{(t+|y|)^{2(n+\varepsilon)}} d y d t\right\}^{1 / 2}\left\|f_{h}^{j}\right\|_{L^{1}\left(\mathbb{R}^{n}\right)} \\
\leq & C\left\{\int_{0}^{|x|} \int_{\Gamma^{\prime \prime}} \frac{t^{2 \varepsilon-n-1}}{(t+|y|)^{2(n+\varepsilon)}} d y d t\right. \\
& \left.+\int_{|x|}^{|\infty|} \int_{\Gamma^{\prime \prime}} \frac{t^{2 \varepsilon-n-1}}{(t+|y|)^{2(n+\varepsilon)}} d y d t\right\}^{1 / 2}\left\|f_{h}^{j}\right\|_{L^{1}\left(\mathbb{R}^{n}\right)^{.}}
\end{aligned}
$$


Thus, similar to $I I_{1}$, we get

$$
I I_{2} \leq C a^{3 n / 2+\varepsilon}|x|^{-n}\left\|f_{h}^{j}\right\|_{L^{1}\left(\mathbb{R}^{n}\right)}
$$

Combining the estimates of $I, I I$, we obtain

$$
S_{\psi, a}\left(f_{h}^{j}\right)(x) \leq C a^{3 n / 2+\gamma+\varepsilon}|x|^{-n}\left\|f_{h}^{j}\right\|_{L^{1}\left(\mathbb{R}^{n}\right)^{*}}
$$

Therefore, by using the above inequality and Minkowski's inequality, we have

$$
\begin{aligned}
& D_{1} \leq C \sup _{k_{0} \in \mathbb{Z}} 2^{-k_{0} \lambda}\left\{\sum_{k=-\infty}^{k_{0}} 2^{k \alpha p}\right. \\
& \left.\times\left(\sum_{j=-\infty}^{k-3}\left\|\left\{\sum_{h}\left(a^{3 n / 2+\gamma+\varepsilon}|x|^{-n}\left\|f_{h}^{j}\right\|_{L^{1}\left(\mathbb{R}^{n}\right)}\right)^{r}\right\}^{1 / r} \chi_{k}\right\|_{L^{q(\cdot)}\left(\mathbb{R}^{n}\right)}\right)^{p}\right\}^{1 / p} \\
& \leq C \sup _{k_{0} \in \mathbb{Z}} 2^{-k_{0} \lambda}\left\{\sum_{k=-\infty}^{k_{0}} 2^{(\alpha-n) k p}\left\|\chi_{k}\right\|_{L^{q(\cdot)}}^{p}\left(\mathbb{R}^{n)}\left(\sum_{j=-\infty}^{k-3}\left\{\sum_{h}\left\|f_{h}^{j}\right\|_{L^{1}\left(\mathbb{R}^{n}\right)}^{r}\right\}^{1 / r}\right)^{p}\right\}^{1 / p}\right. \\
& \leq C \sup _{k_{0} \in \mathbb{Z}} 2^{-k_{0} \lambda}\left\{\sum_{k=-\infty}^{k_{0}} 2^{(\alpha-n) k p}\left\|\chi_{k}\right\|_{L^{q(\cdot)}\left(\mathbb{R}^{n)}\right.}^{p}\left(\sum_{j=-\infty}^{k-3}\left\|\left\{\sum_{h}\left|f_{h}^{j}\right|^{r}\right\}^{1 / r}\right\|_{L^{1}\left(\mathbb{R}^{n}\right)}\right)^{p}\right\}^{1 / p} .
\end{aligned}
$$

It follows from Hölder's inequality and Lemmas 2.2-2.3 that

$$
\begin{aligned}
& D_{1} \leq C \sup _{k_{0} \in \mathbb{Z}} 2^{-k_{0} \lambda}\left\{\sum_{k=-\infty}^{k_{0}} 2^{(\alpha-n) k p}\left\|\chi_{k}\right\|_{L^{q(\cdot)}\left(\mathbb{R}^{n}\right)}^{p}\right. \\
& \left.\times\left(\sum_{j=-\infty}^{k-3}\left\|\left\{\sum_{h}\left|f_{h}^{j}\right|^{r}\right\}^{1 / r}\right\|_{L^{q(\cdot)}\left(\mathbb{R}^{n}\right)}\left\|\chi_{B_{j}}\right\|_{L^{q^{\prime}(\cdot)\left(\mathbb{R}^{n}\right)}}\right)^{p}\right\}^{1 / p} \\
& \leq C \sup _{k_{0} \in \mathbb{Z}} 2^{-k_{0} \lambda}\left\{\sum_{k=-\infty}^{k_{0}} 2^{(\alpha-n) k p}\right. \\
& \left.\times\left\{\sum_{j=-\infty}^{k-3} \frac{\left\|\chi_{B_{j}}\right\|_{L^{q^{\prime}(\cdot)}\left(\mathbb{R}^{n}\right)}}{\left\|\chi_{B_{k}}\right\|_{L^{q^{\prime} \cdot(\cdot)}\left(\mathbb{R}^{n}\right)}}\left|B_{k}\right|\left\|\left\{\sum_{h}\left|f_{h}^{j}\right|^{r}\right\}^{1 / r}\right\|_{L^{q(\cdot)}\left(\mathbb{R}^{n}\right)}\right\}^{p}\right\}^{1 / p} \\
& \leq C \sup _{k_{0} \in \mathbb{Z}} 2^{-k_{0} \lambda}\left\{\sum_{k=-\infty}^{k_{0}} 2^{\alpha k p}\left\{\sum_{j=-\infty}^{k-3}\left(\frac{\left|B_{j}\right|}{\left|B_{k}\right|}\right)^{\delta_{1}}\left\|\left\{\sum_{h}\left|f_{h}^{j}\right|^{r}\right\}^{1 / r}\right\|_{L^{q(\cdot)}\left(\mathbb{R}^{n}\right)}\right\}^{p}\right\}^{1 / p} .
\end{aligned}
$$

Noting that $2^{j \alpha}\left\|\left\{\sum_{h}\left|f_{h}^{j}\right|^{r}\right\}^{1 / r}\right\|_{L^{q(\cdot)}\left(\mathbb{R}^{n}\right)} \leq\left\{\sum_{l=-\infty}^{j} 2^{\alpha l p}\left\|\left\{\sum_{h}\left|f_{h}^{l}\right|^{r}\right\}^{1 / r}\right\|_{L^{q(\cdot)}}^{p}\left(\mathbb{R}^{n}\right\}^{1 / p}\right.$, thus, when $\alpha<\lambda+n \delta_{1}$, we get

$$
\begin{aligned}
D_{1} \leq & C \sup _{k_{0} \in \mathbb{Z}}\left\{\sum _ { k = - \infty } ^ { k _ { 0 } } 2 ^ { \lambda ( k - k _ { 0 } ) p } \left\{\sum_{j=-\infty}^{k-3} 2^{(j-k)\left(n \delta_{1}+\lambda-\alpha\right)}\right.\right. \\
& \left.\left.\times 2^{-j \lambda}\left(\sum_{l=-\infty}^{j} 2^{\alpha l p}\left\|\left\{\sum_{h}\left|f_{h}^{l}\right|^{r}\right\}^{1 / r}\right\|_{L^{q(\cdot)}\left(\mathbb{R}^{n}\right)}^{p}\right)^{1 / p}\right\}^{p}\right\}^{1 / p}
\end{aligned}
$$




$$
\begin{aligned}
& \leq C \sup _{k_{0} \in \mathbb{Z}}\left\{\sum_{k=-\infty}^{k_{0}} 2^{\lambda\left(k-k_{0}\right) p}\left(\sum_{j=-\infty}^{k-3} 2^{(j-k)\left(n \delta_{1}+\lambda-\alpha\right)}\right)^{p}\right\}^{1 / p}\left\|\left\{\sum_{h}\left|f_{h}\right|^{r}\right\}^{1 / r}\right\|_{M \dot{K}_{p, q(\cdot)}^{\alpha, \lambda}\left(\mathbb{R}^{n}\right)} \\
& \leq C\left\|\left\{\sum_{h}\left|f_{h}\right|^{r}\right\}^{1 / r}\right\|_{M \dot{K}_{p, q(\cdot)}^{\alpha, \lambda}\left(\mathbb{R}^{n)}\right.} \cdot
\end{aligned}
$$

Finally, let us to estimate $D_{3}$. By using Minkowski's inequality, we obtain

$$
\begin{aligned}
S_{\psi, a}\left(f_{h}^{j}\right)(x)= & \left(\int_{\Gamma_{a}(x)}\left|\int_{\mathbb{R}^{n}} t^{-n} \psi\left(\frac{y-z}{t}\right) f_{h}^{j}(z) d z\right|^{2} \frac{d y d t}{t^{n+1}}\right)^{1 / 2} \\
\leq & \int_{\mathbb{R}^{n}}\left|f_{h}^{j}(z)\right|\left(\int_{\Gamma_{a}(x)} t^{-3 n-1}\left|\psi\left(\frac{y-z}{t}\right)\right|^{2} d y d t\right)^{1 / 2} d z \\
\leq & \int_{\mathbb{R}^{n}}\left|f_{h}^{j}(z)\right|\left(\int_{0}^{\infty} \int_{\Gamma^{\prime}} t^{-3 n-1}\left|\psi\left(\frac{y-z}{t}\right)\right|^{2} d y d t\right)^{1 / 2} d z \\
& +\int_{\mathbb{R}^{n}}\left|f_{h}^{j}(z)\right|\left(\int_{0}^{\infty} \int_{\Gamma^{\prime \prime}} t^{-3 n-1}\left|\psi\left(\frac{y-z}{t}\right)\right|^{2} d y d t\right)^{1 / 2} d z .
\end{aligned}
$$

Let $a \geq 1, x \in C_{k}, j \geq k+3, z \in C_{j} \subset B_{j}$. Then we have $t+|y-z| \geq \frac{|x-y|}{a}+|y-z| \geq \frac{|x-y|+|y-z|}{a} \geq$ $\frac{|z|-|x|}{a} \geq \frac{3|z|}{4 a}$. Hence, it follows from the condition (ii) that

$$
\begin{aligned}
& \int_{0}^{\infty} \int_{\Gamma^{\prime}} t^{-3 n-1}\left|\psi\left(\frac{y-z}{t}\right)\right|^{2} d y d t \\
& \quad \leq \int_{0}^{\infty} \int_{\Gamma^{\prime}} \frac{t^{2 \varepsilon-n-1}}{(t+|y-z|)^{2(n+\varepsilon)}} d y d t \\
& \quad \leq \int_{0}^{2^{j+1}} \int_{\Gamma^{\prime}} \frac{t^{2 \varepsilon-n-1}}{(t+|y-z|)^{2(n+\varepsilon)}} d y d t+\int_{2^{j+1}}^{\infty} \int_{\Gamma^{\prime}} \frac{t^{2 \varepsilon-n-1}}{(t+|y-z|)^{2(n+\varepsilon)}} d y d t \\
& \leq C \frac{a^{2(n+\varepsilon)}}{|z|^{2(n+\varepsilon)}} \int_{0}^{2^{j+1}} \int_{|x-y|<a t} t^{2 \varepsilon-n-1} d y d t+\int_{2^{j+1}}^{\infty} \int_{y \leq j^{j+1}} t^{-3 n-1} d y d t \\
& \leq C a^{3 n+2 \varepsilon} 2^{-2 j n} .
\end{aligned}
$$

If $y>2^{j+1}$, then $t>\frac{|x-y|}{a} \geq \frac{|x|-|y|}{a}>\frac{2^{j+1}-2^{k}}{a} \geq \frac{2^{j}}{a}$. Applying the condition (ii), we obtain

$$
\begin{aligned}
& \int_{0}^{\infty} \int_{\Gamma^{\prime \prime}} t^{-3 n-1}\left|\psi\left(\frac{y-z}{t}\right)\right|^{2} d y d t \\
& \quad \leq C \int_{\frac{2 j}{a}}^{\infty} \int_{\Gamma^{\prime \prime}} t^{-3 n-1}\left(1+\frac{|y-z|}{t}\right)^{-2(n+\varepsilon)} d y d t \\
& \quad \leq C \int_{\frac{2 j}{a}}^{\infty} \int_{|x-y|<a t} \frac{t^{2 \varepsilon-n-1}}{(t+|y-z|)^{-2(n+\varepsilon)}} d y d t \\
& \leq C a^{3 n} 2^{-2 j n} .
\end{aligned}
$$

Thus

$$
S_{\psi, a}\left(f_{h}^{j}\right)(x) \leq \int_{\mathbb{R}^{n}}\left|f_{h}^{j}(z)\right|\left(C a^{3 n+2 \varepsilon} 2^{-2 j n}+C a^{3 n} 2^{-2 j n}\right)^{1 / 2} d z \leq C a^{3 n / 2+\varepsilon} 2^{-j n}\left\|f_{h}^{j}\right\|_{L^{1}\left(\mathbb{R}^{n}\right)} .
$$


Therefore, similar to $D_{1}$, as $\lambda-n \delta_{2}<\alpha$, by applying Minkowski's inequality, Lemmas 2.12.3, and $2^{j \alpha}\left\|\left\{\sum_{h}\left|f_{h}^{j}\right|^{r}\right\}^{1 / r}\right\|_{L^{q(\cdot)}\left(\mathbb{R}^{n)}\right.} \leq\left\{\sum_{l=-\infty}^{j} 2^{\alpha l p}\left\|\left\{\sum_{h}\left|f_{h}^{l}\right|^{r}\right\}^{1 / r}\right\|_{L^{q(\cdot)}\left(\mathbb{R}^{n}\right)}^{p}\right\}^{1 / p}$, we have

$$
\begin{aligned}
& D_{3} \leq C \sup _{k_{0} \in \mathbb{Z}} 2^{-k_{0} \lambda}\left\{\sum_{k=-\infty}^{k_{0}} 2^{k \alpha p}\left(\sum_{j=k+3}^{\infty}\left\|\left\{\sum_{h}\left(a^{3 n / 2+\varepsilon} 2^{-j n}\left\|f_{h}^{j}\right\|_{L^{1}\left(\mathbb{R}^{n}\right)}\right)^{r}\right\}^{1 / r} \chi_{k}\right\|_{L^{q(\cdot)}\left(\mathbb{R}^{n}\right)}\right)^{p}\right\}^{1 / p} \\
& \leq C \sup _{k_{0} \in \mathbb{Z}} 2^{-k_{0} \lambda}\left\{\sum_{k=-\infty}^{k_{0}} 2^{\alpha k p}\left\|\chi_{k}\right\|_{L^{q(\cdot)}\left(\mathbb{R}^{n)}\right)}^{p}\left(\sum_{j=k+3}^{\infty} 2^{-j n}\left\|\left\{\sum_{h}\left|f_{h}^{j}\right|^{r}\right\}^{1 / r}\right\|_{L^{1}\left(\mathbb{R}^{n)}\right.}\right)^{p}\right\}^{1 / p} \\
& \leq C \sup _{k_{0} \in \mathbb{Z}} 2^{-k_{0} \lambda}\left\{\sum_{k=-\infty}^{k_{0}} 2^{\alpha k p}\left\{\sum_{j=k+3}^{\infty} 2^{-j n}\left|B_{j}\right|\left\|\chi_{B_{k}}\right\|_{L^{q(\cdot)}\left(\mathbb{R}^{n}\right)}\left\|\left\{\sum_{B_{j}} \|_{L^{q(\cdot)}\left(\mathbb{R}^{n}\right)}\left|f_{h}^{j}\right|^{r}\right\}^{1 / r}\right\|_{L^{q(\cdot)}\left(\mathbb{R}^{n}\right)}\right\}^{p}\right\}^{1 / p} \\
& \leq C \sup _{k_{0} \in \mathbb{Z}} 2^{-k_{0} \lambda}\left\{\sum_{k=-\infty}^{k_{0}} 2^{\alpha k p}\left\{\sum_{j=k+3}^{\infty}\left(\frac{\left|B_{k}\right|}{\left|B_{j}\right|}\right)^{\delta_{2}}\left\|\left\{\sum_{h}\left|f_{h}^{j}\right|^{r}\right\}^{1 / r}\right\|_{L^{q(\cdot)}\left(\mathbb{R}^{n}\right)}\right\}^{p}\right\}^{1 / p} \\
& \leq C \sup _{k_{0} \in \mathbb{Z}}\left\{\sum _ { k = - \infty } ^ { k _ { 0 } } 2 ^ { \lambda ( k - k _ { 0 } ) p } \left\{\sum_{j=k+3}^{\infty} 2^{(j-k)\left(-n \delta_{2}+\lambda-\alpha\right)}\right.\right. \\
& \left.\left.\times 2^{-j \lambda}\left(\sum_{l=-\infty}^{j} 2^{\alpha l p}\left\|\left\{\sum_{h}\left|f_{h}^{l}\right|^{r}\right\}^{1 / r}\right\|_{L^{q(\cdot)}\left(\mathbb{R}^{n}\right)}^{p}\right)^{1 / p}\right\}^{p}\right\}^{1 / p} \\
& \leq C\left\|\left\{\sum_{h}\left|f_{h}\right|^{r}\right\}^{1 / r}\right\|_{M \dot{K}_{p, q(\cdot)}^{\alpha, \lambda}\left(\mathbb{R}^{n}\right)} \text {. }
\end{aligned}
$$

Adding up the results of $D_{1}, D_{2}, D_{3}$, we have

$$
\left\|\left\{\sum_{h}\left|S_{\psi, a}\left(f_{h}\right)\right|^{r}\right\}^{1 / r}\right\|_{M \dot{K}_{p, q \cdot(\cdot)}^{\alpha, \lambda}\left(\mathbb{R}^{n)}\right.} \leq C\left\|\left\{\sum_{h}\left|f_{h}\right|^{r}\right\}^{1 / r}\right\|_{M \dot{K}_{p, q(\cdot)}^{\alpha, \lambda}\left(\mathbb{R}^{n}\right)} .
$$

That is, the inequality (1) in Theorem 3.1 holds.

Next we show the other two vector-valued inequalities also hold.

We consider $g_{\psi}$ first. Similar to $S_{\psi, a}$, via calculation, we shall get the following conclusions (also see [22]):

(1) If $x \in C_{k}, j \leq k-3, \operatorname{supp} f_{h}^{j} \subset C_{j}$, then $g_{\psi}\left(f_{h}^{j}\right)(x) \leq C|x|^{-n}\left\|f_{h}^{j}\right\|_{L^{1}\left(\mathbb{R}^{n}\right)}$;

(2) If $x \in C_{k}, j \geq k+3, \operatorname{supp} f_{h}^{j} \subset C_{j}$, then $g_{\psi}\left(f_{h}^{j}\right)(x) \leq C 2^{-j n} \mid f_{h}^{j} \|_{L^{1}\left(\mathbb{R}^{n}\right)}$.

Thus, with a similar argument in the proof of the inequality (1) in Theorem 3.1, by Lemma 2.8 and the above two conclusions, we shall get the following inequality immediately:

$$
\left\|\left\{\sum_{h}\left|g_{\psi}\left(f_{h}\right)\right|^{r}\right\}^{1 / r}\right\|_{M \dot{K}_{p, q(\cdot)}^{\alpha, \lambda}\left(\mathbb{R}^{n}\right)} \leq C\left\|\left\{\sum_{h}\left|f_{h}\right|^{r}\right\}^{1 / r}\right\|_{M \dot{K}_{p, q(\cdot)}^{\alpha, \lambda}\left(\mathbb{R}^{n}\right)} .
$$

For $g_{\mu}^{*}$, by the definition of $S_{\psi, a}$ and $g_{\mu}^{*}$, we have

$$
\begin{aligned}
g_{\mu}^{*}(f)(x) & =\left(\int_{0}^{\infty} \int_{\mathbb{R}^{n}}\left(\frac{t}{t+|x-y|}\right)^{\mu n}\left|\psi_{t} * f(y)\right|^{2} \frac{d y d t}{t^{n+1}}\right)^{1 / 2} \\
& \leq\left(\int_{0}^{\infty} \int_{|x-y|<t}\left(\frac{t}{t+|x-y|}\right)^{\mu n}\left|\psi_{t} * f(y)\right|^{2} \frac{d y d t}{t^{n+1}}\right)^{1 / 2}
\end{aligned}
$$




$$
\begin{aligned}
& +\sum_{l=1}^{\infty}\left(\int_{0}^{\infty} \int_{2^{l-1} t \leq|x-y|<2^{l} t}\left(\frac{t}{t+|x-y|}\right)^{\mu n}\left|\psi_{t} * f(y)\right|^{2} \frac{d y d t}{t^{n+1}}\right)^{1 / 2} \\
\leq & S_{\psi, a}\left(f_{h}^{j}\right)(x)+\sum_{l=1}^{\infty}\left(1+2^{l-1}\right)^{-\mu n / 2} S_{\psi, 2^{l} a}\left(f_{h}^{j}\right)(x) .
\end{aligned}
$$

By the above estimate of $S_{\psi, a}$, we know that, as $x \in C_{k}, j \leq k-3, \operatorname{supp} f_{h}^{j} \subset C_{j}$,

$$
S_{\psi, a}\left(f_{h}^{j}\right)(x) \leq C a^{3 n / 2+\varepsilon+\gamma}|x|^{-n}\left\|f_{h}^{j}\right\|_{L^{1}\left(\mathbb{R}^{n}\right)} .
$$

Thus, when $\mu>3+2(\varepsilon+\gamma) / n$, we have

$$
\begin{aligned}
g_{\mu}^{*}\left(f_{h}^{j}\right)(x) & \leq C a^{3 n / 2+\varepsilon+\gamma}\left(1+\sum_{l=1}^{\infty} 2^{(3 n / 2+\varepsilon+\gamma-\mu n / 2)}\right)|x|^{-n}\left\|f_{h}^{j}\right\|_{L^{1}\left(\mathbb{R}^{n}\right)} \\
& \leq C a^{3 n / 2+\varepsilon+\gamma}|x|^{-n}\left\|f_{h}^{j}\right\|_{L^{1}\left(\mathbb{R}^{n}\right)} .
\end{aligned}
$$

On the other hand, as $x \in C_{k}, j \geq k+3, \operatorname{supp} f_{h}^{j} \subset C_{j}$, we have

$$
S_{\psi, a}\left(f_{h}^{j}\right)(x) \leq C a^{3 n / 2+\varepsilon} 2^{-j n}\left\|f_{h}^{j}\right\|_{L^{1}\left(\mathbb{R}^{n}\right)} .
$$

Furthermore, when $\mu>3+2(\varepsilon+\gamma) / n$, we obtain

$$
\begin{aligned}
g_{\mu}^{*}\left(f_{h}^{j}\right)(x) & \leq C a^{3 n / 2+\varepsilon}\left(1+\sum_{l=1}^{\infty} 2^{(3 n / 2+\varepsilon-\mu n / 2)}\right) 2^{-j n}\left\|f_{h}^{j}\right\|_{L^{1}\left(\mathbb{R}^{n}\right)} \\
& \leq C a^{3 n / 2+\varepsilon} 2^{-j n}\left\|f_{h}^{j}\right\|_{L^{1}\left(\mathbb{R}^{n}\right)^{.}}
\end{aligned}
$$

Hence, similar to the proof of the inequality (1) too, by Lemma 2.8 and the above two conclusions about $g_{\mu}^{*}$, we get the following inequality immediately:

$$
\left\|\left\{\sum_{h}\left|g_{\mu}^{*}\left(f_{h}\right)\right|^{r}\right\}^{1 / r}\right\|_{M \dot{K}_{p, q(\cdot)}^{\alpha, \lambda}\left(\mathbb{R}^{n)}\right.} \leq C\left\|\left\{\sum_{h}\left|f_{h}\right|^{r}\right\}^{1 / r}\right\|_{M \dot{K}_{p, q(\cdot)}^{\alpha, \lambda}\left(\mathbb{R}^{n}\right)} .
$$

This completes the proof of Theorem 3.1.

Now, let us to establish the vector-valued inequalities of the commutators generated by the Littlewood-Paley operators with BMO functions on $M \dot{K}_{p, q(\cdot)}^{\alpha, \lambda}\left(\mathbb{R}^{n}\right)$.

Theorem 3.2 Suppose that $\psi \in L^{1}\left(\mathbb{R}^{n}\right)$ satisfies (i)-(iii), $b \in B M O\left(\mathbb{R}^{n}\right), q(\cdot) \in \mathcal{B}\left(\mathbb{R}^{n}\right), m \in$ $\mathbb{N} \backslash\{0\}$. Let $0<p<\infty, 1<r<\infty, \lambda-n \delta_{2}<\alpha<\lambda+n \delta_{1}$, where $\delta_{1}, \delta_{2}$ is the constant in Lemma 2.2. Then for all function sequence $\left\{f_{h}\right\}_{h=1}^{\infty}:\left\|\left\{\sum_{h}\left|f_{h}\right|^{r}\right\}^{1 / r}\right\|_{M \dot{K}_{p, q(\cdot)}^{\alpha, \lambda}\left(\mathbb{R}^{n}\right)}<\infty$, the following vector-valued inequalities hold:

(1) $\left\|\left\{\sum_{h}\left|\left[b^{m} S_{\psi, a}\right]\left(f_{h}\right)\right|^{r}\right\}^{1 / r}\right\|_{M \dot{K}_{p, q(\cdot)}^{\alpha, \lambda}\left(\mathbb{R}^{n}\right)} \leq C\|b\|_{*}^{m}\left\|\left\{\sum_{h}\left|f_{h}\right|^{r}\right\}^{1 / r}\right\|_{M \dot{K}_{p, q(\cdot)}^{\alpha, \lambda}\left(\mathbb{R}^{n}\right)^{\prime}}$,

(2) $\left\|\left\{\sum_{h}\left|\left[b^{m}, g_{\psi}\right]\left(f_{h}\right)\right|^{r}\right\}^{1 / r}\right\|_{M \dot{K}_{\left.p, q()^{\alpha}\right)}^{\alpha, \lambda}\left(\mathbb{R}^{n}\right)} \leq C\|b\|_{*}\left\|\left\{\sum_{h}\left|f_{h}\right|^{r}\right\}^{1 / r}\right\|_{M \dot{K}_{p, q(\cdot)}^{\alpha, \lambda}\left(\mathbb{R}^{n}\right)^{\prime}}$,

(3) $\left\|\left\{\sum_{h}\left|\left[b^{m}, g_{\mu}^{*}\right]\left(f_{h}\right)\right|^{r}\right\}^{1 / r}\right\|_{M \dot{K}_{p, q(\cdot)}^{\alpha, \lambda}\left(\mathbb{R}^{n}\right)} \leq C\|b\|_{*}^{m}\left\|\left\{\sum_{h}\left|f_{h}\right|^{r}\right\}^{1 / r}\right\|_{M \dot{K}_{p, q(\cdot)}^{\alpha, \lambda}\left(\mathbb{R}^{n}\right)}$, 
where $\mu>3+2(\varepsilon+\gamma) / n, 0<\gamma<\min \{(\mu-2) n / 2, \varepsilon\}$, and the constant $C$ is independent of $\left\{f_{h}\right\}_{h=1}^{\infty}$.

Proof Firstly, we consider the inequality (1). Let $b \in B M O\left(\mathbb{R}^{n}\right)$. For any function sequence $\left\{f_{h}\right\}_{h}$ satisfies \|\|$\left\{f_{h}\right\}_{h}\left\|_{l^{r}}\right\|_{M \dot{K}_{p, q(\cdot)}^{\alpha, \lambda}\left(\mathbb{R}^{n}\right)}<\infty$. We write

$$
f_{h}(x)=\sum_{j=-\infty}^{\infty} f_{h}(x) \chi_{j}(x) \triangleq \sum_{j=-\infty}^{\infty} f_{h}^{j}(x) .
$$

Thus,

$$
\begin{aligned}
& \left\|\left\{\sum_{h}\left|\left[b^{m}, S_{\psi, a}\right]\left(f_{h}\right)\right|^{r}\right\}^{1 / r}\right\|_{M \dot{K}_{p, q(\cdot)}^{\alpha, \lambda}\left(\mathbb{R}^{n)}\right.} \\
& =\sup _{k_{0} \in \mathbb{Z}} 2^{-k_{0} \lambda}\left\{\sum_{k=-\infty}^{k_{0}} 2^{k \alpha p}\left\|\left\{\sum_{h}\left|\left[b^{m}, S_{\psi, a}\right]\left(f_{h}\right)\right|^{r}\right\}^{1 / r} \chi_{k}\right\|_{L^{q(\cdot)}\left(\mathbb{R}^{n}\right)}^{p}\right\}^{1 / p} \\
& \leq \sup _{k_{0} \in \mathbb{Z}} 2^{-k_{0} \lambda}\left\{\sum_{k=-\infty}^{k_{0}} 2^{k \alpha p}\left(\sum_{j=-\infty}^{\infty}\left\|\left\{\sum_{h}\left|\left[b^{m}, S_{\psi, a}\right]\left(f_{h}^{j}\right)\right|^{r}\right\}^{1 / r} \chi_{k}\right\|_{L^{q(\cdot)}\left(\mathbb{R}^{n}\right)}\right)^{p}\right\}^{1 / p} \\
& \leq C \sup _{k_{0} \in \mathbb{Z}} 2^{-k_{0} \lambda}\left\{\sum_{k=-\infty}^{k_{0}} 2^{k \alpha p}\left(\sum_{j=-\infty}^{k-3}\left\|\left\{\sum_{h}\left|\left[b^{m}, S_{\psi, a}\right]\left(f_{h}^{j}\right)\right|^{r}\right\}^{1 / r} \chi_{k}\right\|_{L^{q(\cdot)}\left(\mathbb{R}^{n}\right)}\right)^{p}\right\}^{1 / p} \\
& +C \sup _{k_{0} \in \mathbb{Z}} 2^{-k_{0} \lambda}\left\{\sum_{k=-\infty}^{k_{0}} 2^{k \alpha p}\left(\sum_{j=k-2}^{k+2}\left\|\left\{\sum_{h}\left|\left[b^{m}, S_{\psi, a}\right]\left(f_{h}^{j}\right)\right|^{r}\right\}^{1 / r} \chi_{k}\right\|_{L^{q(\cdot)}\left(\mathbb{R}^{n}\right)}\right)^{p}\right\}^{1 / p} \\
& +C \sup _{k_{0} \in \mathbb{Z}} 2^{-k_{0} \lambda}\left\{\sum_{k=-\infty}^{k_{0}} 2^{k \alpha p}\left(\sum_{j=k+3}^{\infty}\left\|\left\{\sum_{h}\left|\left[b^{m}, S_{\psi, a}\right]\left(f_{h}^{j}\right)\right|^{r}\right\}^{1 / r} \chi_{k}\right\|_{L^{q(\cdot)}\left(\mathbb{R}^{n}\right)}\right)^{p}\right\}^{1 / p} \\
& \triangleq E_{1}+E_{2}+E_{3} .
\end{aligned}
$$

We are now going to estimate each term, respectively. For the term $E_{2}$, noting that $\operatorname{supp} f_{h}^{j} \subset C_{j}$, we can easily obtain by Lemma 2.9

$$
\begin{aligned}
E_{2} & \leq C \sup _{k_{0} \in \mathbb{Z}} 2^{-k_{0} \lambda}\left\{\sum_{k=-\infty}^{k_{0}} 2^{k \alpha p}\left(\sum_{j=k-2}^{k+2}\left\|\left\{\sum_{h}\left|f_{h}^{j}\right|^{r}\right\}^{1 / r}\right\|_{L^{q(\cdot)\left(\mathbb{R}^{n}\right)}}\right)^{p}\right\}^{1 / p} \\
& \leq C \sup _{k_{0} \in \mathbb{Z}} 2^{-k_{0} \lambda}\left\{\sum_{k=-\infty}^{k_{0}} 2^{k \alpha p}\left\|\left\{\sum_{h}\left|f_{h}\right|^{r}\right\}^{1 / r} \chi_{k}\right\|_{L^{q(\cdot)}\left(\mathbb{R}^{n)}\right)}^{p}\right\}^{1 / p} \\
& =C\left\|\left\{\sum_{h}\left|f_{h}\right|^{r}\right\}^{1 / r}\right\|_{M \dot{K}_{p, q(\cdot)}^{\alpha, \lambda}\left(\mathbb{R}^{n)}\right.} .
\end{aligned}
$$

For the term $E_{1}$, observe that as $x \in C_{k}, j \leq k-3, \operatorname{supp} f_{h}^{j} \subset C_{j}$. With the same argument as in the estimate of $D_{1}$, we have

$$
S_{\psi, a}\left(f_{h}^{j}\right)(x) \leq C a^{3 n / 2+\varepsilon+\gamma}|x|^{-n}\left\|f_{h}^{j}\right\|_{L^{1}\left(\mathbb{R}^{n}\right)} .
$$


Wang and Tao Journal of Inequalities and Applications 2014, 2014:227

Page 14 of 17

Thus,

$$
\begin{aligned}
\left|\left[b^{m}, S_{\psi, a}\right]\left(f_{h}^{j}\right)(x)\right| & =\left|S_{\psi, a}\left[(b(x)-b)^{m} f\right](x)\right| \\
& \leq C a^{3 n / 2+\varepsilon+\gamma}|x|^{-n}\left\|(b(\cdot)-b)^{m} f_{h}^{j}\right\|_{L^{1}\left(\mathbb{R}^{n}\right)^{.}}
\end{aligned}
$$

Hence, by Minkowski’s inequality, we get

$$
\begin{gathered}
\left\|\left\{\sum_{h}\left|\left[b^{m}, S_{\psi, a}\right]\left(f_{h}^{j}\right)\right|^{r}\right\}^{1 / r} \chi_{k}\right\|_{L^{q(\cdot)}\left(\mathbb{R}^{n}\right)} \\
\leq C\left\||x|^{-n}\left\{\sum_{h}\left\|(b(\cdot)-b)^{m} f_{h}^{j}\right\|_{L^{1}\left(\mathbb{R}^{n}\right)}^{r}\right\}^{1 / r} \chi_{k}\right\|_{L^{q(\cdot)}\left(\mathbb{R}^{n}\right)} \\
\leq C\left\||x|^{-n}\right\|\left\{\sum_{h}\left|(b(\cdot)-b)^{m} f_{h}^{j}\right|^{r}\right\}^{1 / r}\|\|_{L^{1}\left(\mathbb{R}^{n}\right)} \chi_{k} \|_{L^{q(\cdot)}\left(\mathbb{R}^{n}\right)} \\
\leq C 2^{-n k}\left\|\left(b-b_{j}\right)^{m} \chi_{k}\right\|_{L^{q(\cdot)\left(\mathbb{R}^{n}\right)} \|}\left\|\left\{\sum_{h}\left|f_{h}^{j}\right|^{r}\right\}^{1 / r}\right\|_{L^{1}\left(\mathbb{R}^{n}\right)} \\
+2^{-n k}\left\|\chi_{k}\right\|_{L^{q(\cdot)}\left(\mathbb{R}^{n}\right)}\left\|\left\{\sum_{h}\left|\left(b-b_{j}\right)^{m} f_{h}^{j}\right|^{r}\right\}^{1 / r}\right\|_{L^{1}\left(\mathbb{R}^{n}\right)} .
\end{gathered}
$$

Furthermore, by Hölder's inequality and Lemmas 2.2-2.4, we obtain

$$
\begin{aligned}
& \left\|\left\{\sum_{h}\left|\left[b^{m}, S_{\psi, a}\right]\left(f_{h}^{j}\right)\right|^{r}\right\}^{1 / r} \chi_{k}\right\|_{L^{q(\cdot)}\left(\mathbb{R}^{n}\right)} \\
& \leq C 2^{-n k}(k-j)^{m}\|b\|_{*}^{m}\left\|\chi_{B_{k}}\right\|_{L^{q(\cdot)}\left(\mathbb{R}^{n}\right)}\left\|\left\{\sum_{h}\left|f_{h}^{j}\right|^{r}\right\}^{1 / r}\right\|_{L^{q(\cdot)}\left(\mathbb{R}^{n}\right)}\left\|\chi_{j}\right\|_{L^{q^{\prime}(\cdot)}\left(\mathbb{R}^{n}\right)} \\
& +2^{-n k}\left\|\chi_{B_{k}}\right\|_{L^{q(\cdot)}\left(\mathbb{R}^{n}\right)}\left\|\left\{\sum_{h}\left|f_{h}^{j}\right|^{r}\right\}^{1 / r}\right\|_{L^{q(\cdot)}\left(\mathbb{R}^{n}\right)}\left\|\left(b-b_{j}\right)^{m} \chi_{j}\right\|_{L^{q^{\prime}(\cdot)}\left(\mathbb{R}^{n}\right)} \\
& \leq C 2^{-n k}\left[(k-j)^{m}+1\right]\|b\|_{*}^{m}\left\|\chi_{B_{k}}\right\|_{L^{q(\cdot)}\left(\mathbb{R}^{n}\right)}\left\|\left\{\sum_{h}\left|f_{h}^{j}\right|^{r}\right\}^{1 / r}\right\|_{L^{q(\cdot)}\left(\mathbb{R}^{n}\right)}\left\|\chi_{B_{j}}\right\|_{L^{q^{\prime}(\cdot)}\left(\mathbb{R}^{n}\right)} \\
& \leq C 2^{-n k}(k-j)^{m}\|b\|_{*}^{m}\left|B_{k}\right| \frac{\left\|\chi_{B_{j}}\right\|_{L^{q^{\prime}(\cdot)}\left(\mathbb{R}^{n}\right)}}{\left\|\chi_{B_{k}}\right\|_{L^{q^{\prime} \cdot(\cdot)}\left(\mathbb{R}^{n}\right)}}\left\|\left\{\sum_{h}\left|f_{h}^{j}\right|^{r}\right\}^{1 / r}\right\|_{L^{q(\cdot)}\left(\mathbb{R}^{n}\right)} \\
& \leq C(k-j)^{m}\|b\|_{*}^{m} \frac{\left|B_{j}\right|}{\left|B_{k}\right|}\left\|\left\{\sum_{h}\left|f_{h}^{j}\right|^{r}\right\}^{1 / r}\right\|_{L^{q(\cdot)}\left(\mathbb{R}^{n}\right)} \\
& \leq C 2^{(j-k) n \delta_{1}}(k-j)^{m}\|b\|_{*}^{m}\left\|\left\{\sum_{h}\left|f_{h}^{j}\right|^{r}\right\}^{1 / r}\right\|_{L^{q(\cdot)}\left(\mathbb{R}^{n}\right)} .
\end{aligned}
$$

Therefore, as $\alpha<\lambda+n \delta_{1}$, noting that

$$
2^{j \alpha}\left\|\left\{\sum_{h}\left|f_{h}^{j}\right|^{r}\right\}^{1 / r}\right\|_{L^{q(\cdot)}\left(\mathbb{R}^{n}\right)} \leq\left\{\sum_{l=-\infty}^{j} 2^{\alpha l p}\left\|\left\{\sum_{h}\left|f_{h}^{l}\right|^{r}\right\}^{1 / r}\right\|_{L^{q(\cdot)}\left(\mathbb{R}^{n)}\right.}^{p}\right\}^{1 / p},
$$


then we have

$$
\begin{aligned}
E_{1} \leq & C\|b\|_{*}^{m} \sup _{k_{0} \in \mathbb{Z}} 2^{-\lambda k_{0}}\left\{\sum_{k=-\infty}^{k_{0}} 2^{\alpha k p}\left(\sum_{j=-\infty}^{k-3} 2^{(j-k) n \delta_{1}}(k-j)^{m}\left\|\left\{\sum_{h}\left|f_{h}^{j}\right|^{r}\right\}^{1 / r}\right\|_{L^{q \cdot \cdot\left(\mathbb{R}^{n}\right)}}\right)^{p}\right\}^{1 / p} \\
\leq & C\|b\|_{*}^{m} \sup _{k_{0} \in \mathbb{Z}}\left\{\sum _ { k = - \infty } ^ { k _ { 0 } } 2 ^ { \lambda ( k - k _ { 0 } ) p } \left(\sum_{j=-\infty}^{k-3}(k-j)^{m} 2^{(j-k)\left(n \delta_{1}+\lambda-\alpha\right)}\right.\right. \\
& \left.\left.\left.\times 2^{-j \lambda}\left\{\sum_{l=-\infty}^{j} 2^{\alpha l p}\left\|\left\{\sum_{h}\left|f_{h}^{l}\right|^{r}\right\}^{1 / r}\right\|_{L^{q \cdot \cdot(\cdot)}}^{p}\right\}^{n}\right)^{1 / p}\right)^{p}\right\}^{1 / p} \\
\leq & C\|b\|_{*}^{m}\left\|\left\{\sum_{h}\left|f_{h}\right|^{r}\right\}^{1 / r}\right\|
\end{aligned}
$$

Now we turn to estimate $E_{3}$. Observe that as $x \in C_{k}, j \geq k+3$, supp $f_{h}^{j} \subset C_{j}$. With the same argument as in the estimate of $D_{3}$, then we have

$$
S_{\psi, a}\left(f_{h}^{j}\right)(x) \leq C a^{3 n / 2+\varepsilon} 2^{-j n}\left\|f_{h}^{j}\right\|_{L^{1}\left(\mathbb{R}^{n}\right)} .
$$

Thus,

$$
\left|\left[b^{m}, S_{\psi, a}\right]\left(f_{h}^{j}\right)(x)\right| \leq C a^{3 n / 2+\varepsilon} 2^{-j n}\left\|(b(\cdot)-b)^{m} f_{h}^{j}\right\|_{L^{1}\left(\mathbb{R}^{n}\right)} .
$$

Hence, by Minkowski's inequality, we get

$$
\begin{gathered}
\left\|\left\{\sum_{h}\left|\left[b^{m}, S_{\psi, a}\right]\left(f_{h}^{j}\right)\right|^{r}\right\}^{1 / r} \chi_{k}\right\|_{L^{q(\cdot)}\left(\mathbb{R}^{n}\right)} \\
\leq C\left\|2^{-j n}\left\{\sum_{h}\left\|(b(\cdot)-b)^{m} f_{h}^{j}\right\|_{L^{1}\left(\mathbb{R}^{n}\right)}^{r}\right\}^{1 / r} \chi_{k}\right\|_{L^{q(\cdot)}\left(\mathbb{R}^{n}\right)} \\
\leq C\left\|2^{-j n}\right\|\left\{\sum_{h}\left|(b(\cdot)-b)^{m} f_{h}^{j}\right|^{r}\right\}^{1 / r}\left\|_{L^{1}\left(\mathbb{R}^{n}\right)} \chi_{k}\right\|_{L^{q(\cdot)}\left(\mathbb{R}^{n}\right)} \\
\leq C 2^{-j n}\left\|\left(b-b_{k}\right)^{m} \chi_{k}\right\|_{L^{q(\cdot)}\left(\mathbb{R}^{n}\right)}\left\|\left\{\sum_{h}\left|f_{h}^{j}\right|^{r}\right\}^{1 / r}\right\|_{L^{1}\left(\mathbb{R}^{n}\right)} \\
\quad+2^{-j n}\left\|\chi_{k}\right\|_{L^{q(\cdot)}\left(\mathbb{R}^{n}\right)}\left\|\left\{\sum_{h}\left|\left(b-b_{k}\right)^{m} f_{h}^{j}\right|^{r}\right\}^{1 / r}\right\|_{L^{1}\left(\mathbb{R}^{n}\right)} .
\end{gathered}
$$

Furthermore, by Hölder's inequality and Lemmas 2.2-2.4, we obtain

$$
\begin{aligned}
& \left\|\left\{\sum_{h}\left|\left[b^{m}, S_{\psi, a}\right]\left(f_{h}^{j}\right)\right|^{r}\right\}^{1 / r} \chi_{k}\right\|_{L^{q(\cdot)}\left(\mathbb{R}^{n}\right)}
\end{aligned}
$$

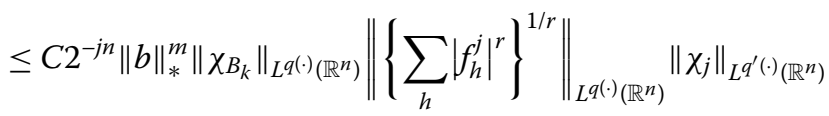

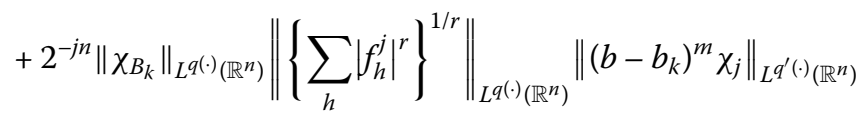




$$
\begin{aligned}
& \leq C 2^{-j n}\left[(k-j)^{m}+1\right]\|b\|_{*}^{m}\left\|\chi_{B_{k}}\right\|_{L^{q \cdot(\cdot)}\left(\mathbb{R}^{n)}\right)}\left\|\left\{\sum_{h}\left|f_{h}^{j}\right|^{r}\right\}^{1 / r}\right\|_{L^{q(\cdot)}\left(\mathbb{R}^{n}\right)}\left\|\chi_{B_{j}}\right\|_{L^{q^{\prime} \cdot(\cdot)}} \mathbb{R}^{n)} \\
& \leq C 2^{-j n}(k-j)^{m}\|b\|_{*}^{m}\left|B_{j}\right| \frac{\left\|\chi_{B_{k}}\right\|_{L^{q(\cdot)}\left(\mathbb{R}^{n}\right)}}{\left\|\chi_{B_{j}}\right\|_{L^{q(\cdot)}\left(\mathbb{R}^{n}\right)}}\left\|\left\{\sum_{h}\left|f_{h}^{j}\right|^{r}\right\}^{1 / r}\right\|_{L^{q(\cdot)}\left(\mathbb{R}^{n)}\right.} \\
& \leq C(k-j)^{m}\|b\|_{*}^{m} \frac{\left|B_{k}\right|}{\left|B_{j}\right|}\left\|\left\{\sum_{h}\left|f_{h}^{j}\right|^{r}\right\}^{1 / r}\right\|_{L^{q(\cdot)\left(\mathbb{R}^{n}\right)}} \\
& \leq C 2^{(k-j) n \delta_{2}}(j-k)^{m}\|b\|_{*}^{m}\left\|\left\{\sum_{h}\left|f_{h}^{j}\right|^{r}\right\}^{1 / r}\right\|_{L^{q(\cdot)\left(\mathbb{R}^{n}\right)}} .
\end{aligned}
$$

Therefore, similar to $E_{1}$, as $\alpha>\lambda-n \delta_{2}$, noting that

$$
2^{j \alpha}\left\|\left\{\sum_{h}\left|f_{h}^{j}\right|^{r}\right\}^{1 / r}\right\|_{L^{q(\cdot)}\left(\mathbb{R}^{n}\right)} \leq\left\{\sum_{l=-\infty}^{j} 2^{\alpha l p}\left\|\left\{\sum_{h}\left|f_{h}^{l}\right|^{r}\right\}^{1 / r}\right\|_{L^{q(\cdot)}\left(\mathbb{R}^{n)}\right.}^{p}\right\}^{1 / p},
$$

then we have

$$
\begin{aligned}
E_{3} \leq & C\|b\|_{*}^{m} \sup _{k_{0} \in \mathbb{Z}} 2^{-\lambda k_{0}}\left\{\sum_{k=-\infty}^{k_{0}} 2^{\alpha k p}\left(\sum_{j=k+3}^{\infty} 2^{(k-j) n \delta_{2}}(j-k)^{m}\left\|\left\{\sum_{h}\left|f_{h}^{j}\right|^{r}\right\}^{1 / r}\right\|_{L^{q \cdot(\cdot)}\left(\mathbb{R}^{n}\right)}\right)^{p}\right\}^{1 / p} \\
\leq & C\|b\|_{*}^{m} \sup _{k_{0} \in \mathbb{Z}}\left\{\sum _ { k = - \infty } ^ { k _ { 0 } } 2 ^ { \lambda ( k - k _ { 0 } ) p } \left(\sum_{j=k+3}^{\infty}(j-k)^{m} 2^{(j-k)\left(-n \delta_{2}+\lambda-\alpha\right)}\right.\right. \\
& \left.\left.\left.\times 2^{-j \lambda}\left\{\sum_{l=-\infty}^{j} 2^{\alpha l p}\left\|\left\{\sum_{h}\left|f_{h}^{l}\right|^{r}\right\}^{1 / r}\right\|_{L^{q \cdot \cdot(\cdot)}}^{p}\right\}^{n}\right\}^{1 / p}\right)^{p}\right\}^{1 / p} \\
\leq & C\|b\|_{*}^{m}\left\|\left\{\sum_{h}\left|f_{h}\right|^{r}\right\}^{1 / r}\right\|_{M \dot{K}_{p, q(\cdot)}^{\alpha, \lambda}\left(\mathbb{R}^{n)}\right.} \cdot
\end{aligned}
$$

Adding up the results of $E_{1}, E_{2}, E_{3}$, we have

$$
\left\|\left\{\sum_{h}\left|\left[b^{m} S_{\psi, a}\right]\left(f_{h}\right)\right|^{r}\right\}^{1 / r}\right\|_{M \dot{K}_{p, q(\cdot)}^{\alpha, \lambda}\left(\mathbb{R}^{n}\right)} \leq C\|b\|_{*}^{m}\left\|\left\{\sum_{h}\left|f_{h}\right|^{r}\right\}^{1 / r}\right\|_{M \dot{K}_{p, q(\cdot)}^{\alpha, \lambda}\left(\mathbb{R}^{n}\right)} .
$$

That is, the inequality (1) in Theorem 3.2 holds.

For the proofs about the vector-valued inequalities of $\left[b^{m}, g_{\psi}\right]$ and $\left[b^{m}, g_{\mu}^{*}\right]$, with a similar arguments in the proof of the vector-valued inequality (1) in Theorem 3.2, by Lemma 2.9 and the estimates of $g_{\psi}$, and $g_{\mu}^{*}$ in Theorem 3.1, it is not difficult to deduce

$$
\left\|\left\{\sum_{h}\left|\left[b^{m}, g_{\psi}\right]\left(f_{h}\right)\right|^{r}\right\}^{1 / r}\right\|_{M \dot{K}_{p, q(\cdot)}^{\alpha, \lambda}\left(\mathbb{R}^{n}\right)} \leq C\|b\|_{*}\left\|\left\{\sum_{h}\left|f_{h}\right|^{r}\right\}^{1 / r}\right\|_{M \dot{K}_{p, q(\cdot)}^{\alpha, \lambda}\left(\mathbb{R}^{n}\right)}
$$

and, if $\mu>3+2(\varepsilon+\gamma) / n$ and $0<\gamma<\min \{(\mu-2) n / 2, \varepsilon\}$,

$$
\left\|\left\{\sum_{h}\left|\left[b^{m}, g_{\mu}^{*}\right]\left(f_{h}\right)\right|^{r}\right\}^{1 / r}\right\|_{M \dot{K}_{p, q(\cdot)}^{\alpha, \lambda}\left(\mathbb{R}^{n)}\right.} \leq C\|b\|_{*}^{m}\left\|\left\{\sum_{h}\left|f_{h}\right|^{r}\right\}^{1 / r}\right\|_{M \dot{K}_{p, q(\cdot)}^{\alpha, \lambda}\left(\mathbb{R}^{n}\right)} .
$$

We complete the proof of Theorem 3.2. 
Remark 3.1 By Remark 1.1, we can easily to see that the results in Theorems 3.1-3.2 are also suitable for Herz spaces with variable exponent $\dot{K}_{p(\cdot)}^{\alpha, q}\left(\mathbb{R}^{n}\right)$.

\author{
Competing interests \\ The authors declare that they have no competing interests.
}

Authors' contributions

All authors contributed equally to the writing of this paper. All authors read and approved the final manuscript.

\title{
Acknowledgements
}

Shuangping Tao is supported by National Natural Foundation of China (Grant No. 11161042 and 11071250).

Received: 25 December 2013 Accepted: 23 May 2014 Published: 03 Jun 2014

\section{References}

1. Stein, E: The development of square functions in the work of A. Zygmund. Bull. Am. Math. Soc. (N.S.) 7, 359-376 (1982)

2. Kenig, C: Harmonic Analysis Techniques for Second Order Elliptic Boundary Value Problems. Am. Math. Soc Providence (1994)

3. Chang, S, Wilson, J, Wolff, T: Some weighted norm inequalities concerning the Schrödinger operators. Comment. Math. Helv. 60, 217-246 (1985)

4. Lu, SZ, Yang, DC: The central BMO spaces and Littlewood-Paley operators. Approx. Theory Appl. 11, 72-94 (1995)

5. Zhang, MJ, Liu, LZ: Sharp weighted inequality for multilinear commutator of the Littlewood-Paley operator. Acta Math. Vietnam. 30(2), 181-189(2005)

6. Xue, QY, Ding, Y: Weighted estimates for multilinear commutators of the Littlewood-Paley operators. Sci. China Ser. A 39(3), 315-332 (2009)

7. Wei, XM, Tao, SP: Boundedness for parametrized Littlewood-Paley operators with rough kernels on weighted weak Hardy spaces. Abstr. Appl. Anal. 2013, Article ID 651941 (2013)

8. Wei, XM, Tao, SP: The boundedness of Littlewood-Paley operators with rough kernels on weighted $\left(L^{q}, L^{p}\right)^{\alpha}\left(\mathbb{R}^{n}\right)$ spaces. Anal. Theory Appl. 29(2), 135-148 (2013)

9. Martell, JM: Sharp maximal functions associated with approximations of the identity in spaces of homogeneous type and applications. Stud. Math. 161, 113-145 (2004)

10. Kováčik, O, Rákosník, J: On spaces $L^{p(x)}$ and $W^{k, p(x)}$. Czechoslov. Math. J. 41(116), 592-618 (1991)

11. Acerbi, E, Mingione, G: Gradient estimates for a class of parabolic systems. Duke Math. J. 136, 285-320 (2007)

12. Acerbi, E, Mingione, G: Regularity results for stationary electrorheological fluids. Arch. Ration. Mech. Anal. 164, 213-259 (2002)

13. Diening, L, Ružička, M: Calderón-Zygmund operators on generalized Lebesgue spaces $L^{p(\cdot)}$ and problems related to fluid dynamics. J. Reine Angew. Math. 563, 197-220 (2003)

14. Ružička, M: Electrorheological Fluids: Modeling and Mathematical Theory. Lecture Notes in Math., vol. 1748. Springer, Berlin (2000)

15. Zhikov, W: Averaging of functionals of the calculus of variations and elasticity theory. Izv. Akad. Nauk SSSR, Ser. Mat. 50, 675-710 (1986) (in Russian)

16. Cruz-Uribe, D, Fiorenza, A, Martell, JM, Pérez, C: The boundedness of classical operators on variable $L^{p}$ spaces. Ann. Acad. Sci. Fenn., Math. 31, 239-264 (2006)

17. Karlovich, $A Y$, Lerner, AK: Commutators of singular integrals on generalized $L^{P}$ spaces with variable exponent. Publ. Mat. 49, 111-125 (2005)

18. Izuki, M: Fractional integrals on Herz-Morrey spaces with variable exponent. Hiroshima Math. J. 40, 343-355 (2010)

19. Izuki, M: Boundedness of vector-valued sublinear operators on Herz-Morrey spaces with variable exponent. Math. Sci. Res. J. 13, 243-253 (2009)

20. Izuki, M: Boundedness of commutators on Herz spaces with variable exponent. Rend. Circ. Mat. Palermo 59(2), 199-213 (2010)

21. Nekvinda, A: Hardy-Littlewood maximal operator on $L^{p(x)}\left(\mathbb{R}^{n}\right)$. Math. Inequal. Appl. 7, $255-265$ (2004)

22. Lu, SZ, Yang, DC, Hu, GE: Herz Type Spaces and Their Applications, pp. 131-132. Science Press, Beijing (2008)

10.1186/1029-242X-2014-227

Cite this article as: Wang and Tao: Boundedness of Littlewood-Paley operators and their commutators on Herz-Morrey spaces with variable exponent. Journal of Inequalities and Applications 2014, 2014:227 